\title{
Mental health in UK Biobank: development, implementation and results from an online questionnaire completed by 157366 participants - CORRIGENDUM
}

Katrina A. S. Davis, Jonathan R. I. Coleman, Mark Adams, Naomi Allen, Gerome Breen, Breda Cullen, Chris Dickens, Elaine Fox, Nick Graham, Jo Holliday, Louise M. Howard, Ann John, William Lee, Rose McCabe, Andrew Mclntosh, Robert Pearsall, Daniel J. Smith, Cathie Sudlow, Joey Ward, Stan Zammit and Matthew Hotopf

\section{Copyright and usage}

(c) The Royal College of Psychiatrists 2018. This is an Open Access article, distributed under the terms of the creative Commons Attribution-NonCommercial-ShareAlike licence (http://creativecommons.org/licenses/by-nc-sa/4.0/), which permits non-commercial re-use, distribution, and reproduction in any medium, provided the same Creative Commons licence is included and the original work is properly cited. The written permission of Cambridge University Press must be obtained for commercial re-use.

https://doi.org/10.1192/bjo.2018.12, Published by Cambridge University Press, $3^{\text {rd }}$ April 2018.

It has recently come to our attention that the above paper contains an error. One of the variables in table 3 labelled "moderate activity $\geq$ three times a week", and also referred to in the text as "Achieving recommended levels of physical activity", was extracted incorrectly.
Please see below an updated version of table 3 with the changes highlighted. The numbers of participants who are positive for this measure is much reduced, but our conclusion that there was no difference between the groups is unchanged.

The authors apologise for this error.

\section{Reference}

Davis K., Coleman J., Adams M., Allen N., Breen G., Cullen B., ... Hotopf M. (2018). Mental health in UK Biobank: Development, implementation and results from an online questionnaire completed by 157366 participants. BJPsych Open, 4(3), 8390. doi:10.1192/bjo.2018.12 
Table 3 Socioeconomic factors by status for lifetime occurrence (people may be included in more than one category). See lettered table notes, and Appendix 2 for full case definitions

\begin{tabular}{|c|c|c|c|c|c|c|c|}
\hline & & $\begin{array}{l}\text { No "lifetime" } \\
\text { criteria met }^{a} \\
\mathrm{~N}=102,901\end{array}$ & $\begin{array}{l}\text { Depression }^{b} \\
\mathrm{~N}=37,434\end{array}$ & $\begin{array}{l}\text { Bipolar type } 1^{\mathrm{c}} \\
\mathrm{N}=931\end{array}$ & $\begin{array}{l}\text { Anxiety disorder } \\
(G A D)^{b} \\
N=11,111\end{array}$ & $\begin{array}{l}\text { Unusual } \\
\text { experiences }^{d} \\
\mathrm{~N}=7,803\end{array}$ & $\begin{array}{l}\text { Addiction }^{\mathrm{e}} \\
\mathrm{N}=9,386\end{array}$ \\
\hline \multicolumn{8}{|c|}{ Personal Characteristics } \\
\hline \multirow[t]{4}{*}{ Age $^{f}$} & $45-54$ & 14364 (13\%) & 7145 (19\%) & $228(24 \%)$ & $2348(21 \%)$ & 1485 (19\%) & 2013 (21\%) \\
\hline & $55-64$ & 33307 (31\%) & $14809(40 \%)$ & $417(45 \%)$ & $4470(40 \%)$ & 2904 (37\%) & 3428 (37\%) \\
\hline & $65-74$ & 51705 (48\%) & 13739 (37\%) & $261(28 \%)$ & 3892 (35\%) & $2960(38 \%)$ & 3466 (37\%) \\
\hline & $75+$ (oldest is 82) & $9376(9 \%)$ & $1741(5 \%)$ & 25 (3\%) & $401(4 \%)$ & 454 (6\%) & 479 (5\%) \\
\hline Gender & female & $57556(53 \%)$ & 25815 (69\%) & 532 (57\%) & $7404(67 \%)$ & $4718(60 \%)$ & 4556 (49\%) \\
\hline Ethnicity & white & 105072 (97\%) & 36297 (97\%) & 892 (96\%) & 10749 (97\%)c & $7503(96 \%)$ & 9037 (96\%) \\
\hline $\begin{array}{l}\text { Townsend } \\
\text { Deprivation } \\
\text { Score }^{g}\end{array}$ & $\begin{array}{l}\text { most deprived } \\
\qquad(T D S \geq+2)\end{array}$ & 11783 (11\%) & $5656(15 \%)$ & $201(22 \%)$ & $1856(17 \%)$ & $1426(18 \%)$ & 1941 (21\%) \\
\hline $\begin{array}{l}\text { Highest } \\
\text { qualification }\end{array}$ & degree & $48700(45 \%)$ & $16939(45 \%)$ & $425(46 \%)$ & $5071(46 \%)$ & $3646(47 \%)$ & 4531 (48\%) \\
\hline \multicolumn{8}{|l|}{ Known risk factors } \\
\hline Neuroticism score ${ }^{i}$ & mean (SD) & $3.2(2.8)$ & $5.6(3.3)$ & $3.8(3.1)$ & $7.1(3.3)$ & $5.2(3.5)$ & $5.4(3.5)$ \\
\hline \multirow{3}{*}{$\begin{array}{l}\text { Adverse life } \\
\quad \text { experiences }\end{array}$} & childhood screen ${ }^{j}$ & $43913(40 \%)$ & 21144 (56\%) & $638(69 \%)$ & $6931(62 \%)$ & $4783(61 \%)$ & $5800(62 \%)$ \\
\hline & adult screenk ${ }^{k}$ & $50226(46 \%)$ & 23893 (64\%) & 685 (74\%) & 7581 (68\%) & $4783(61 \%)$ & 6303 (67\%) \\
\hline & trauma exposure & 50771 (47\%) & 22166 (59\%) & 665 (71\%) & $6877(62 \%)$ & 5439 (70\%) & $6278(67 \%)$ \\
\hline \multirow[t]{2}{*}{ Social connection } & loneliness ${ }^{i, m}$ & 2976 (3\%) & $2367(6 \%)$ & $94(10 \%)$ & $971(9 \%)$ & $570(7 \%)$ & $669(7 \%)$ \\
\hline & social isolation ${ }^{i}$ & 7793 (7\%) & 3623 (10\%) & $126(14 \%)$ & $1173(11 \%)$ & 931 (12\%) & $1200(13 \%)$ \\
\hline Illness & $\begin{array}{l}\text { longstanding illness, } \\
\text { disability or infirmity' }\end{array}$ & $26341(24 \%)$ & $13363(36 \%)$ & $503(54 \%)$ & 4581 (41\%) & 3242 (42\%) & 3588 (38\%) \\
\hline \multicolumn{8}{|l|}{ Health-behaviours } \\
\hline \multirow[t]{3}{*}{ Smoking status ${ }^{i}$} & current & $6235(6 \%)$ & 3638 (10\%) & 158 (17\%) & $1194(11 \%)$ & 837 (11\%) & 1916 (20\%) \\
\hline & former & 36425 (33\%) & 13927 (37\%) & 323 (35\%) & 4009 (36\%) & $2943(38 \%)$ & 4893 (52\%) \\
\hline & never & $65827(61 \%)$ & $19786(53 \%)$ & 448 (48\%) & $5883(53 \%)$ & $4003(51 \%)$ & 2547 (27\%) \\
\hline \multirow{3}{*}{$\begin{array}{l}\text { Cannabis use } \\
\text { (lifetime) }\end{array}$} & daily & $868(1 \%)$ & $914(2 \%)$ & $63(7 \%)$ & $346(3 \%)$ & $258(3 \%)$ & 867 (9\%) \\
\hline & ever, but not daily & 19675 (18\%) & 9607 (26\%) & 299 (32\%) & $2818(25 \%)$ & $2312(30 \%)$ & 3487 (37\%) \\
\hline & never & 88209 (81\%) & $26913(72 \%)$ & 569 (61\%) & 7947 (72\%) & $5233(67 \%)$ & 5032 (54\%) \\
\hline Physical activity & $\begin{array}{l}\text { moderate activity } \geq \\
\text { three times a week }\end{array}$ & 39677 (38\%) & 13988 (38\%) & $345(38 \%)$ & $4174(39 \%)$ & $2846(38 \%)$ & 3602 (39\%) \\
\hline \multicolumn{8}{|c|}{$\begin{array}{l}\text { a) Criteria not met for depression, GAD, unusual experiences or addiction. } \\
\text { b) Criteria met for disorder on CIDI-SF lifetime. } \\
\text { c) Criteria met for both lifetime depression and lifetime mania. } \\
\text { d) Reported potential hallucination or delusion at any point in their life. } \\
\text { e) Positively endorsed: "Have you been addicted to or dependent on one or more things, including substances (not cigarettes/coffee) or behaviours (such as gambling)?" } \\
\text { f) Age when mental health questionnaire released, derived from date of birth. } \\
\text { g) Townsend material Deprivation Score is based on postcode areas. } \\
\text { h) Collapsed from categories in table } 2 \text {. } \\
\text { i) From baseline assessment } 2007-10 \text {. } \\
\text { j) Criteria met for possible abuse or neglect on Childhood Trauma Screener. } \\
\text { k) Criteria met for adverse situations as an adult: lack of confiding relationship, abusive relationships and money problems. } \\
\text { l) Reports one or more of six situations that are known to be triggers for trauma-related disorders. } \\
\text { m) There is some overlap between the adult screen and loneliness screen, which both ask about confiding relationships: adult screen includes lack of confiding relationship over the adult } \\
\text { lifetime; loneliness includes lack of confiding relationship at the time of baseline assessment. }\end{array}$} \\
\hline
\end{tabular}

